\title{
Lipólisis láser y liposucción en ginecomastia: retracción cutánea eficaz y segura
}

\section{Laser-assisted lipolysis for gynecomastia: safe and effective skin retraction}

\author{
Mario Trelles ${ }^{1,2}$; Enrique Bonanad ${ }^{1,2}$; Javier Moreno-Moraga 3 ; Justo Alcolea ${ }^{3}$; Serge Mordon ${ }^{4}$; Franck Marie LeClère ${ }^{4}$
}

\author{
R E S U M E N
}

\begin{abstract}
Objetivo: Valorar la eficacia de la lipólisis-láser para corregir el volumen, flacidez y excedente cutáneo sin escisión. Métodos: Prospectivamente, en 32 pacientes con ginecomastia, bajo anestesia tumescente y sedación se realizó lipólisis con láser de diodo 980nm, 15W en emisión continua, 8kJ a 12kJ de energía por mama. Externamente se utilizó aire frío para protección de la piel. Después se utilizó lipoaspiración convencional. No se emplearon drenajes pero si vendaje compresivo. Los pacientes evaluaron los resultados en una escala visual analógica. Dos médicos evaluaron los resultados por fotografías de antes y seis meses después, y midieron las areolas y contorno torácico. Resultados: Veintitrés pacientes consideraron los resultados como Muy Bueno, siete Bueno y dos Regular. La retracción cutánea en la areola fue notable un mes después de la intervención y fue máxima a los seis meses. La valoración de los médicos fue 26 Muy Bueno, cinco Bueno y un Regular. No existieron quemaduras, isquemia, ni lesiones en areolas o pezón. Conclusión: La liposucción asistida por láser es eficaz y de ejecución sencilla, poco traumática y permite una temprana reintegración a las actividades.
\end{abstract}

Palabras clave: Rayos láser. Lipólisis. Láseres de semiconductores. Lipectomía. Ginecomastia.

\section{INTRODUCCIÓN}

D esde la primera descripción sobre ginecomastia por Aegineta', han seguido diversos estudios sobre las varias etiologías ligadas a su desarrollo². Igualmente, para su tratamiento se han comunicado numerosas técnicas de escisión y liposucción ${ }^{3,4}$. En el momento actual conocemos que en un rango comprendido entre el $32 \mathrm{y}$ el $65 \%$, los hombres padecen feminización de las mamas $^{5}$. A la clasificación clínica de las ginecomastias por Simon ${ }^{6}$, se han añadido otras clasificaciones ${ }^{7}$ y recomendaciones sobre técnicas de tratamiento ${ }^{8,9}$. En todas ellas se presta especial atención a los métodos de eliminación de la piel redundante, conjuntamente con la reducción del diámetro de la areola. Este detalle adquiere máxima importancia cuando se intenta conseguir un diseño estético que cumpla con el patrón anatómico, tanto en las dimensiones y volumen de la mama, como con las relaciones del complejo areolapezón y las proporciones de la región pectoral. En la valoración del resultado del tratamiento de la ginecomastia el exceso de piel y la reconstrucción de la región pectoral determinan el éxito quirúrgico, el resultado estético y la satisfacción del paciente.

La lipólisis láser acompañada de liposucción convencional es actualmente una técnica bien establecida ${ }^{10}$.
El conocimiento de los detalles quirúrgicos, junto al avance tecnológico experimentado, han permitido progresar eficientemente desde que por primera vez Apfelberg comunicara sobre el tratamiento de la ginecomastia con asistencia del láser ${ }^{11}$. Al incluir la lipólisis láser en el procedimiento de liposucción, aparte de tener una buena tolerancia quirúrgica y ofrecer una rápida recuperación, el efecto que se consigue de contracción de la piel constituye una ventaja importante. La inflamación que se produce en el tejido después del tratamiento láser, ligada al depósito térmico residual por efecto del láser, consigue mejorar la laxitud del tejido en la fase postoperatoria, evitando la escisión del exceso de piel y la necesidad de remodelación de la región músculo pectoral ${ }^{12}$.

Presentamos nuestra experiencia en un análisis prospectivo de una muestra de 32 pacientes diagnosticados de ginecomastia, en los cuales se empleó la lipólisis láser acompañada de liposucción convencional. Este estudio focaliza particularmente en el efecto de contracción de la piel y los resultados conseguidos que remedian la laxitud cutánea y reducen el tamaño de la areola. Estos detalles tienen mucha importancia en la apreciación positiva que los pacientes hacen de los resultados, que dependen en gran medida de la resolución de la piel redundante junto a la reducción del tamaño de la mama.

1. Instituto Médico Vilafortuny. Cambrils, Tarragona, Espana; 2. Fundación Antoni de Gimbernat, Espana; 3. Instituto Médico Láser. Madrid, Espana; 4. INSERM U703, Hospital Universitario Lille, Francia. 


\section{MÉTODOS}

Treinta y dos pacientes de edades comprendidas entre 22 y 64 años (media 38,03 años), que presentaban ginecomastia de más de ocho años de evolución fueron examinados y seleccionados de forma aleatoria para este estudio. Todos firmaron el consentimiento informado después de explicarles los detalles correspondientes a la cirugía y el tipo de cuestionarios que debían completar. El protocolo del estudio prospectivo fue revisado por el Comité Ético del Instituto Médico Vilafortuny, que fue aceptado con la finalidad de confirmar las observaciones previas, sobre el mismo tema, que en su día fueron comunicadas ${ }^{12}$. A todos los pacientes se les realizó estudio clínico para descartar cualquier enfermedad que pudiera tener relación con la ginecomastia que presentaban. Durante el examen se identificó sobrepeso en 13 de los 32 pacientes (Tabla 1). Los exámenes hormonales adicionales practicados para la determinación de testosterona, estradiol, función hepáti$\mathrm{ca}$, renal y tiroidea fueron normales. Las radiografías de tórax y las mamografías no mostraron razones tumorales de relevancia como causa de ginecomastia. Si en algunos de los pacientes se identificó variaciones de alguna de las pruebas referidas ${ }^{13}$, fueron excluidos del estudio y se intervinieron quirúrgicamente, de forma convencional.

De los 32 pacientes, nueve presentaban flacidez cutánea y cinco tenían asimetría del diámetro de las areolas. Teniendo estos detalles en cuenta, se realizó marcaje del área pectoral para definir los límites de la liposucción. El pliegue inferior de la mama se marcó de $2 \mathrm{~cm}$ a $3 \mathrm{~cm}$ por debajo de su localización normal. También se definieron los puntos de incisión para introducir la cánula de infiltración anestésica, que serían los mismos para introducir la cánula con la fibra láser. Posteriormente, las mismas incisiones se emplearon para introducir la cánula de aspiración de la grasa (Figura 1)

Tabla 1 - Características de los pacientes.

\begin{tabular}{|c|c|c|c|c|c|}
\hline $\mathrm{N}$ & Edad & Sobrepeso & Flacidez cutánea & $\begin{array}{l}\text { Asimetría de la } \\
\text { posición de la areola }\end{array}$ & $\begin{array}{c}\text { Body Mass } \\
\text { Index }\end{array}$ \\
\hline 1 & 35 & Si & $\mathrm{Si}$ & No & 32.0 \\
\hline 2 & 22 & $\mathrm{Si}$ & No & $\mathrm{Si}$ & 34.5 \\
\hline 3 & 41 & $\mathrm{Si}$ & $\mathrm{Si}$ & No & 36.0 \\
\hline 4 & 38 & $\mathrm{Si}$ & No & No & 33.6 \\
\hline 5 & 64 & No & No & No & 22.3 \\
\hline 6 & 29 & No & No & $\mathrm{Si}$ & 20.0 \\
\hline 7 & 35 & No & No & No & 21.8 \\
\hline 8 & 58 & No & No & No & 21.0 \\
\hline 9 & 37 & No & No & No & 23.0 \\
\hline 10 & 38 & $\mathrm{Si}$ & $\mathrm{Si}$ & No & 32.1 \\
\hline 11 & 29 & $\mathrm{Si}$ & $\mathrm{Si}$ & No & 33.4 \\
\hline 12 & 42 & No & No & No & 22.7 \\
\hline 13 & 48 & No & No & $\mathrm{Si}$ & 22.4 \\
\hline 14 & 25 & No & No & No & 21.7 \\
\hline 15 & 38 & $\mathrm{Si}$ & No & No & 34.2 \\
\hline 16 & 39 & No & No & No & 22.2 \\
\hline 17 & 38 & No & No & No & 21.6 \\
\hline 18 & 37 & No & No & No & 20.0 \\
\hline 19 & 33 & No & No & No & 21.3 \\
\hline 20 & 31 & No & No & No & 22.5 \\
\hline 21 & 37 & $\mathrm{Si}$ & $\mathrm{Si}$ & No & 33.8 \\
\hline 22 & 29 & $\mathrm{Si}$ & $\mathrm{Si}$ & No & 28.8 \\
\hline 23 & 37 & No & No & No & 20.7 \\
\hline 24 & 46 & $\mathrm{Si}$ & $\mathrm{Si}$ & No & 36.6 \\
\hline 25 & 38 & No & No & $\mathrm{Si}$ & 22.0 \\
\hline 26 & 23 & $\mathrm{Si}$ & No & No & 31.3 \\
\hline 27 & 52 & $\mathrm{Si}$ & $\mathrm{Si}$ & $\mathrm{Si}$ & 29.4 \\
\hline 28 & 48 & No & $\mathrm{Si}$ & No & 20.9 \\
\hline 29 & 28 & $\mathrm{Si}$ & No & No & 29.7 \\
\hline 30 & 27 & No & No & No & 21.6 \\
\hline 31 & 50 & No & No & No & 20.2 \\
\hline 32 & 45 & No & No & No & 20.8 \\
\hline
\end{tabular}



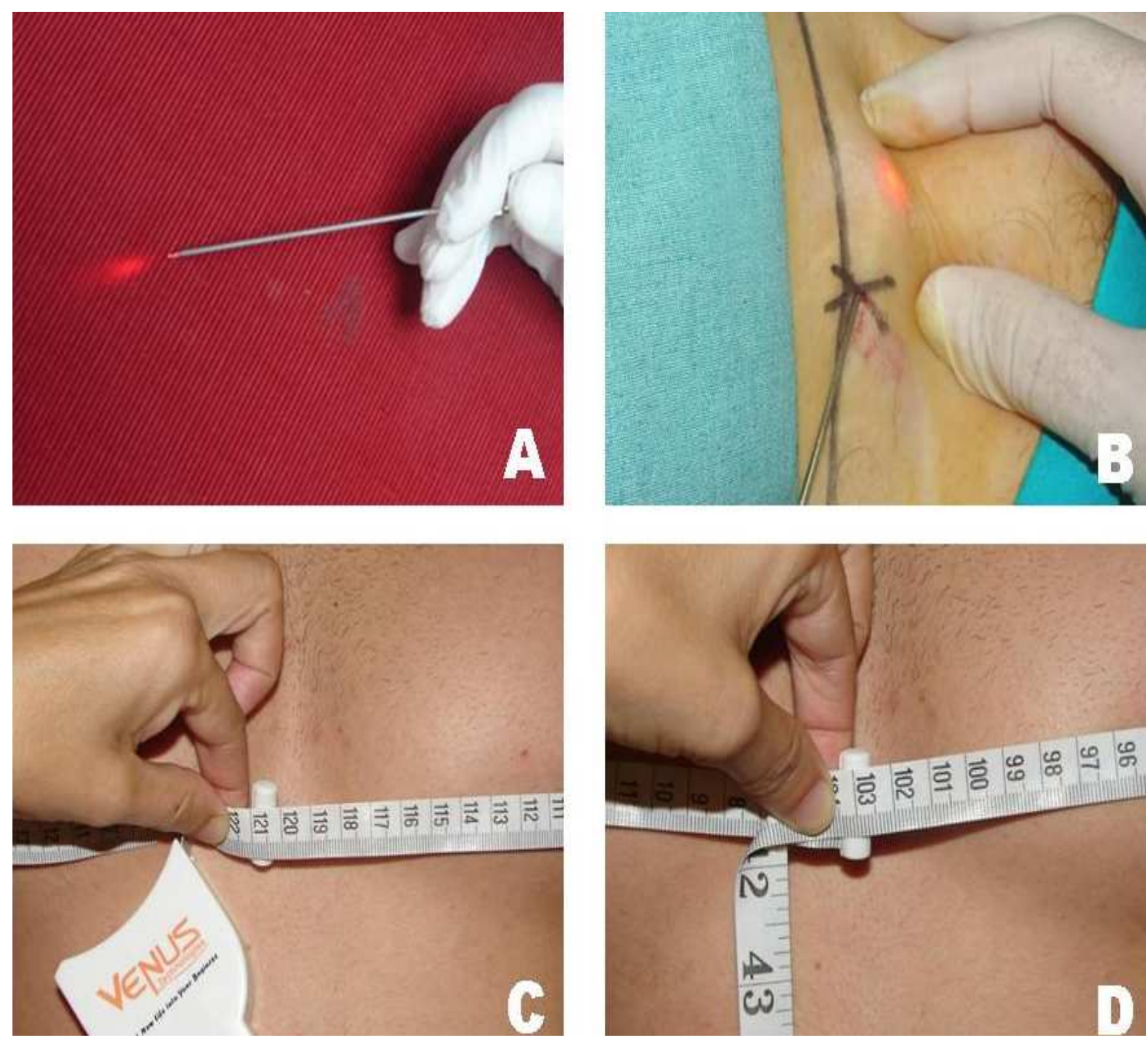

Figura 1 - A) La punta de la fibra sobresale aproximadamente $5 \mathrm{~mm}$. La luz guía del láser permite seguir la posición en que se produce la irradiación. B) Se observa la luz guía de láser a través de la piel durante la lipólisis. C y D corresponden a las mediadas del tórax de antes y seis meses después, respectivamente, del paciente \#5.

En todos los pacientes se realizó anestesia tumescente empleando una cánula roma multiperforada de $12 \mathrm{~cm}$ de longitud y $5 \mathrm{~mm}$ de diámetro, y una jeringa de $20 \mathrm{ml}$. La mezcla de anestesia fue de lidocaína al 0,1\% con epinefrina 1:1.000.000, siguiendo la fórmula de Klein ${ }^{14}$. Se inyectaron entre $350 \mathrm{ml} \mathrm{y} 500 \mathrm{ml}$ de anestesia por mama y todos los pacientes fueron sedados con midazolan por el anestesiólogo. Aproximadamente 15 minutos después de la inyección anestésica tumescente se practicaron dos incisiones de $2 \mathrm{~mm}$ con una hoja de bisturí no 11 , a fin de dar paso a una cánula de $9 \mathrm{~cm}$ de longitud y $1 \mathrm{~mm}$ de diámetro para efectuar las maniobras de tunelización. En los casos de mamas cónicas con fibrosis dura del tejido mamario se empleó una cánula de Toledo para estas maniobras. Inmediatamente después se introdujo, a través de la misma cánula empleada para tunelización, la fibra óptica de 600 micras para conducir la luz láser. La punta de la fibra excedió aproximadamente $5 \mathrm{~mm}$ la salida de la cánula (Figura 1). El otro extremo de la fibra se conectó a un láser de Diodo de 980nm de longitud de onda.

Los parámetros empleados fueron $15 \mathrm{~W}$ de potencia en emisión continua. El tiempo de exposición de la radiación láser se relacionó con el volumen de la mama. La cantidad de energía total acumulada por cada mama fue entre $8 \mathrm{~kJ}$ a 12kJ (kiloJulios). La irradiación láser siguió el plano de la cánula, realizando un movimiento cuidadoso hacia adelante y atrás de forma continua. El paso de la cánula se hizo en dos planos del tejido graso, comenzando 
por un plano más profundo. Al pasar al plano superficial, más cercano a la piel, el movimiento se realizó más rápidamente, procurando que el choque de la punta de la fibra con la piel fuera suave. La palma de la mano se situó por encima de la zona de actuación del láser para detectar y controlar la posición de la punta de la fibra. El propósito de tocar la piel con el movimiento de la cánula fue dirigir hacia la dermis el efecto térmico láser de una forma lo más homogénea posible. Se tuvo cuidado de no lesionar con la fibra la piel superficial, coordinando la emisión láser y el movimiento de la cánula de forma regular. Mientras el láser emitía su energía se tuvo cuidado de no detener el vaivén de la cánula, a fin de evitar quemar la piel, sobre todo debajo del complejo areola-pezón y no lesionar el pedículo vascular. Si el caso correspondió a una ginecomastia asimétrica, se aumentó el número de pases de la cánula, irradiando $2 \mathrm{~kJ}$ a $4 \mathrm{~kJ}$ extras en la mama más desarrollada o flácida.

Al mismo tiempo que el láser emitía su energía, la piel se enfrió externamente con un aparato que emite un flujo continuo de aire frío a $-20^{\circ} \mathrm{C}$, hasta 1000 de litros/ minuto. Mientras el láser irradiaba internamente en el tejido, los cambios de temperatura de la piel se siguieron con un termómetro infrarrojo teniendo cuidado de que la temperatura detectada externamente no excediera de $42^{\circ} \mathrm{C}$. En caso de aumento de esta temperatura, la cánula se movió hacia otra zona a fin de prevenir quemaduras.

Una vez que la energía láser se distribuyó lo más homogéneamente posible en toda el área de tratamiento, se realizó la lipoaspiración empleando un aparato de succión a un bar de presión negativa. Para esta maniobra se empleó una cánula recta de $1 \mathrm{~mm}$ de diámetro. Tras la aspiración se llevó a cabo el examen del área tratada, colocando la palma de la mano sobre la mama, en la cual previamente se vertió una pequeña cantidad de suero salino con la finalidad de ayudar en su deslizamiento y detectar posibles irregularidades en el tejido. Terminada la exploración, las incisiones se cerraron con una sola grapa metálica por cada incisión. No se colocaron drenajes, y para el vendaje se emplearon compresas plegadas en forma de triángulo, y después una banda ancha elástica. El vendaje fue de tipo compresivo y fue sujetado firmemente, colocando encima una banda torácica, que se indicó mantener durante siete días. Pasado este tiempo se recomendó llevar la compresión 20 días más durante todo el día, aunque podía retirarse para la higiene personal. Transcurrido este tiempo se indicó llevarla solo de noche, durante un mes más.

Como medicación se prescribió prednisolona (30mg/día), durante cuatro días; paracetamol (650mg cada seis horas, cinco días) y amoxicilina $(500 \mathrm{mg})+$ ácido clavulánico (125mg), cada ocho horas, durante seis días.

Seis meses después de la cirugía, para valorar subjetivamente los resultados finales, a todos los pacientes se les solicitó que indicaran en una escala visual analógica, asignando una valoración según la siguiente puntuación:
Muy Bueno, entre 75 y 100; Bueno, entre 50 y 74; Regular, entre 25 y 49, y Malo, entre 0 y 24. Las visitas de revisión de todos los pacientes se realizaron siete días, uno y seis meses después de la cirugía. A todos los pacientes se les solicitó que no valoraran solo aquellos cambios observados en la disminución del volumen o la proyección de las mamas, sino que tuvieran en cuenta los cambios operados en la calidad del tejido y si apreciaban mejoría en la laxitud de la piel y los posibles cambios acontecidos en el diámetro de las areolas. A los pacientes se les facilitó fotografías de antes de la cirugía y se les pidió que realizaran el examen del área tratada frente a un espejo. Un médico de los participantes en el estudio estuvo presente durante el examen, pero solo para ayudar en la interpretación que el paciente debía hacer del aspecto y proyección de la mama, y todos aquellos detalles relacionados con las características de la areola y calidad de la piel.

En la visita previa a la cirugía y en la última visita seis meses después, se pesó a cada paciente y tomaron medidas, con la finalidad de analizar comparativamente cualquier variación. La medición del perímetro pectoral y el diámetro de las areolas se realizó con el paciente estando de pie, empleando una cinta métrica colocada siempre a la misma altura. Se tomó como referencia los pezones y el tórax en espiración. La valoración objetiva, que fue realizada por dos examinadores independientes al estudio, expertos en este tipo de cirugía, se realizó utilizando las mismas puntuaciones que en la valoración subjetiva, teniendo en cuenta el grado de simetría obtenido con respecto a la simetría del tamaño areolar. Con esta finalidad se comprobaron las fotografías de antes y seis meses después de la intervención y las mediciones realizadas.

\section{RESULTADOS}

Aparte de las reacciones propias de la cirugía, como edema, equimosis y pequeños hematomas, en ningún caso fue necesaria su evacuación por estar muy limitados. Ningún paciente presentó complicaciones.

El dolor pudo controlarse bien con Paracetamol, $650 \mathrm{mg}$ cada seis horas. En el examen no se observaron signos de isquemia, congestión de las areolas o quemaduras cutáneas, tampoco se detectaron cambios en la sensibilidad en los pezones. Las grapas metálicas para sutura se retiraron siete días después de la cirugía, sin que se advirtieran infecciones y/o cicatrizaciones anómalas.

En la tabla 2 se recogen los resultados de las valoraciones subjetivas y objetivas a los seis meses de finalizada la cirugía. Las valoraciones de los resultados Muy Bueno y Bueno, sumadas entre ellas, mostraron que el tratamiento fue satisfactorio, para pacientes y médicos, tanto desde un punto de vista estético como en la disminución del diámetro de la areola, la mejoría de la calidad de la piel y la firmeza de la piel de la región pectoral (Figuras 2 a 4). No fue hasta un mes después de la cirugía 
que los pacientes notaron una reducción notable del tamaño de la mama, junto con cambios de la piel que se mostró entonces más firme. La proyección de las mamas y las areolas disminuyeron progresivamente $y$, a los seis meses, 23 pacientes de los 32 tratados puntuaron los resultados como Muy Bueno, siete Bueno, y dos los calificaron como Regular. Estos dos pacientes expresaron que la reducción de las mamas fue insuficiente y que la cirugía no había cumplido con sus expectativas. Ambos pacientes tenían sobrepeso, con índice de masa corporal (BMI) en torno a $30 \mathrm{Kg} / \mathrm{m}^{2}$, lo que indicó la importancia que tiene la correcta selección del paciente para este tipo de cirugía.

La valoración subjetiva que hicieron los pacientes sobre el tamaño, volumen y proyección de las mamas, así como las características de la piel y tamaño de la areola se presentan en la tabla 2. En la valoración objetiva, basada en el examen de las fotografías de antes y seis meses después de la cirugía, y en la medida de la circunferencia del tórax, se encontró una disminución media de $12,5 \mathrm{~cm}$ y entre $1 \mathrm{~cm}$ y $3 \mathrm{~cm}$ (media 1.78) en el diámetro de la areola (Tabla 2). Ninguno de los pacientes presentó una circunferencia de tórax menor de $100 \mathrm{~cm}$ antes de la intervención. Los médicos que examinaron las fotografías valoraron 26 resultados como Muy Bueno; cinco Bueno, y Regular en un solo caso. El grado de mejoría fue progresivo desde la primera visita siete días después de la cirugía, hasta el último control a los seis meses. Se concluyó que la reducción del edema y reafirmación del tejido, que fue progresivo, no debería valorarse antes de los seis meses para saber el verdadero alcance del tratamiento. Un mes después de la cirugía la flacidez, y otros signos propios de un rejuvenecimiento cutáneo no habían resuelto plenamen-

Tabla 2 - Evaluación objetiva y subjetiva a los 6 meses y mediciones del tórax y areola.

\begin{tabular}{|c|c|c|c|c|c|c|c|c|c|}
\hline & Pacien & Valorac & subjetiva & & ediciones ( & & Doctores: & Valoración & Objetiva \\
\hline & $\begin{array}{l}\text { Muy } \\
\text { Bueno }\end{array}$ & Bueno & Regular & $\begin{array}{l}\text { Tórax } \\
\text { Antes }\end{array}$ & $\begin{array}{l}\text { Tórax } \\
\text { Después }\end{array}$ & $\begin{array}{c}\text { Areola } \\
\text { Diámetro }\end{array}$ & $\begin{array}{c}\text { Muy } \\
\text { Bueno }\end{array}$ & Bueno & Regular \\
\hline 1 & $x$ & & & 145 & 122 & 3.3 & $x$ & & \\
\hline 2 & $x$ & & & 115 & 104 & 1.1 & $x$ & & \\
\hline 3 & & $x$ & & 138 & 110 & 2.8 & & $x$ & \\
\hline 4 & & & $x$ & 102 & 98 & 1 & $x$ & & \\
\hline 5 & $x$ & & & 121 & 103 & 1.8 & $x$ & & \\
\hline 6 & & $x$ & & 120 & 115 & 1.4 & $x$ & & \\
\hline 7 & $x$ & & & 122 & 106 & 3 & $x$ & & \\
\hline 8 & $x$ & & & 116 & 105 & 1.2 & $x$ & & \\
\hline 9 & & $x$ & & 128 & 112 & 2.2 & & $x$ & \\
\hline 10 & $x$ & & & 135 & 109 & 2.8 & $x$ & & \\
\hline 11 & $x$ & & & 139 & 114 & 2.1 & $x$ & & \\
\hline 12 & $x$ & & & 102 & 98 & 1.5 & $x$ & & \\
\hline 13 & $x$ & & & 115 & 106 & 1.8 & $x$ & & \\
\hline 14 & $x$ & & & 108 & 97 & 1.6 & $x$ & & \\
\hline 15 & & $x$ & & 136 & 112 & 1.8 & $x$ & & \\
\hline 16 & $x$ & & & 105 & 94 & 1 & $x$ & & \\
\hline 17 & $x$ & & & 121 & 109 & 1.1 & $x$ & & \\
\hline 18 & & $x$ & & 119 & 106 & 1.2 & & $x$ & \\
\hline 19 & $x$ & & & 115 & 108 & 1.6 & $x$ & & \\
\hline 20 & $x$ & & & 118 & 102 & 2 & $x$ & & \\
\hline 21 & $x$ & & & 137 & 126 & 1.8 & $x$ & & \\
\hline 22 & $x$ & & & 135 & 122 & 1.9 & $x$ & & \\
\hline 23 & & $x$ & & 112 & 108 & 1 & & $x$ & \\
\hline 24 & & & $x$ & 144 & 123 & 1.5 & & & $x$ \\
\hline 25 & $x$ & & & 122 & 111 & 3 & $x$ & & \\
\hline 26 & $x$ & & & 138 & 115 & 2.2 & $x$ & & \\
\hline 27 & $x$ & & & 112 & 106 & 1 & $x$ & & \\
\hline 28 & $x$ & & & 100 & 96 & 1.2 & $x$ & & \\
\hline 29 & & $x$ & & 136 & 120 & 2.6 & & $x$ & \\
\hline 30 & $x$ & & & 112 & 107 & 1.2 & $x$ & & \\
\hline 31 & $x$ & & & 118 & 109 & 1.4 & $x$ & & \\
\hline 32 & $x$ & & & 109 & 101 & 1.8 & $x$ & & \\
\hline
\end{tabular}



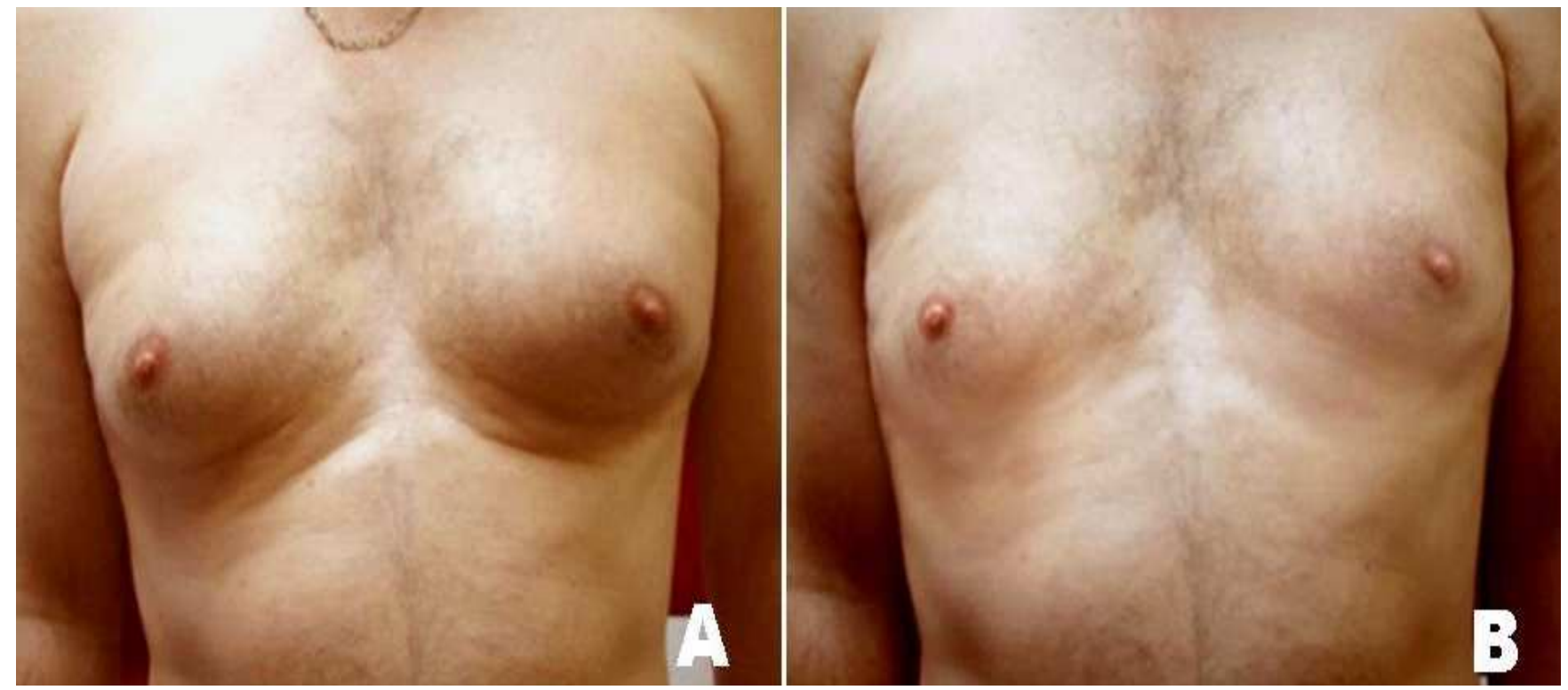

Figura 2 - Paciente \#13. A, antes, nótese la sombra Inframamaria que resalta protección mamaria e incomodaba al paciente. No existe piel laxa ni el paciente presentaba sobrepeso. B, seis meses después. Reducción notable del volumen de las mamas y corrección de la sombra inframamaria. El resultado fue valorado como $M B$, con notable retracción cutánea y disminución del diámetro de la areola.
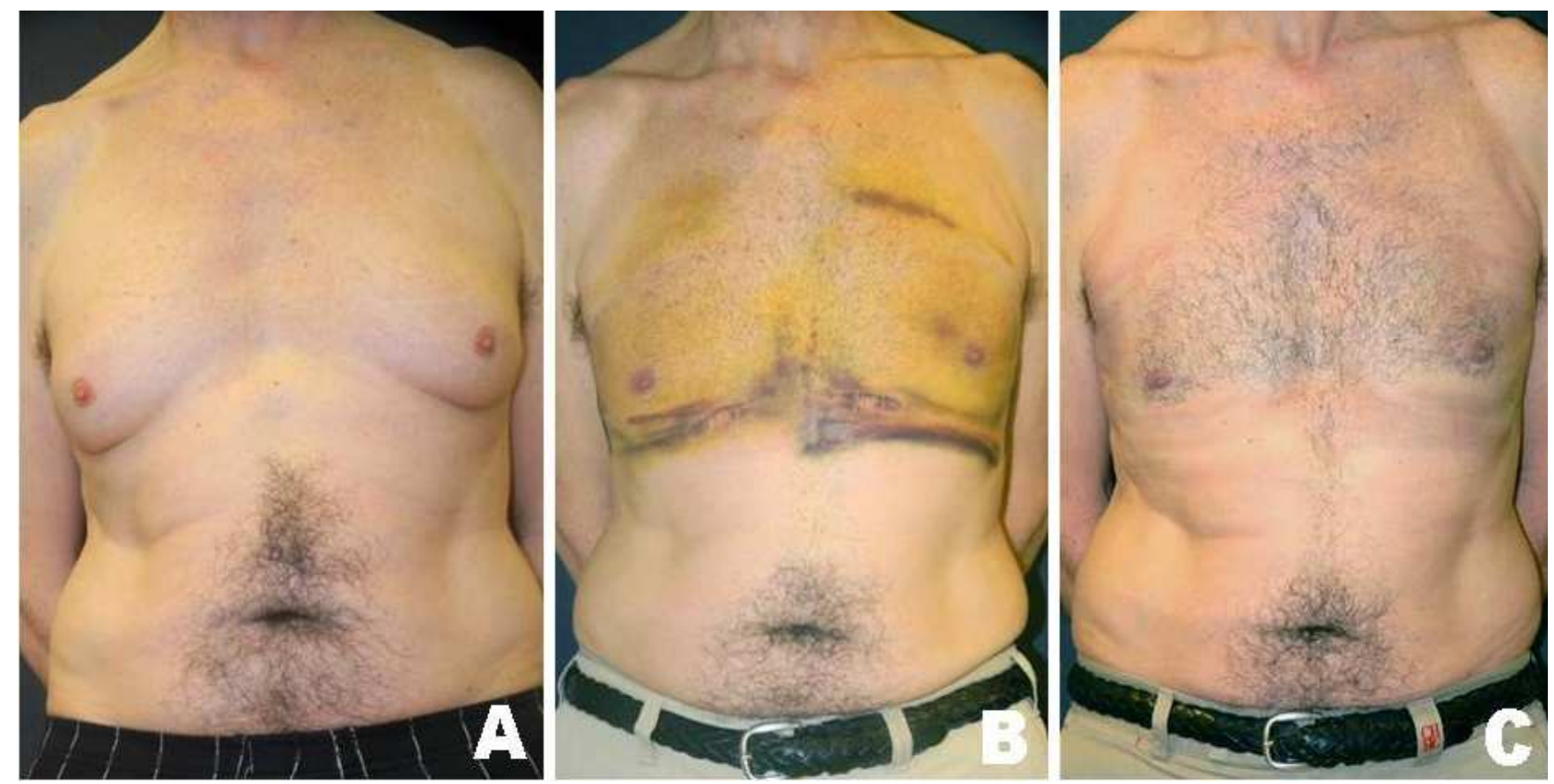

Figura 3 - Paciente \#27; A, antes y B, una semana después al retirar el vendaje compresivo; Se observa equimosis. La reducción del volumen mamario de la areola es notoria. C, muestra los resultados (MB), seis meses después de la cirugía. Obsérvese la evidente retracción cutánea. La reducción del perímetro toráxico es de $6 \mathrm{~cm}$ y de $1 \mathrm{~cm}$ en el diámetro de la areola.

te, y el tejido continuó mejorando progresivamente según se observó en el último control efectuado a los seis meses. La disminución del diámetro de la areola y la firmeza obtenida en la piel fue entonces más notable. La disminución del diámetro de las areolas, de todos los casos, expresados en centímetros, se presentan en la tabla 2.

\section{DISCUSION}

Desde la primera comunicación sobre tratamientos combinados para ginecomastia ${ }^{15}$, varios logros han hecho posible despejar el camino hacia el éxito y hacia una cirugía más depurada de alcance óptimo. La 


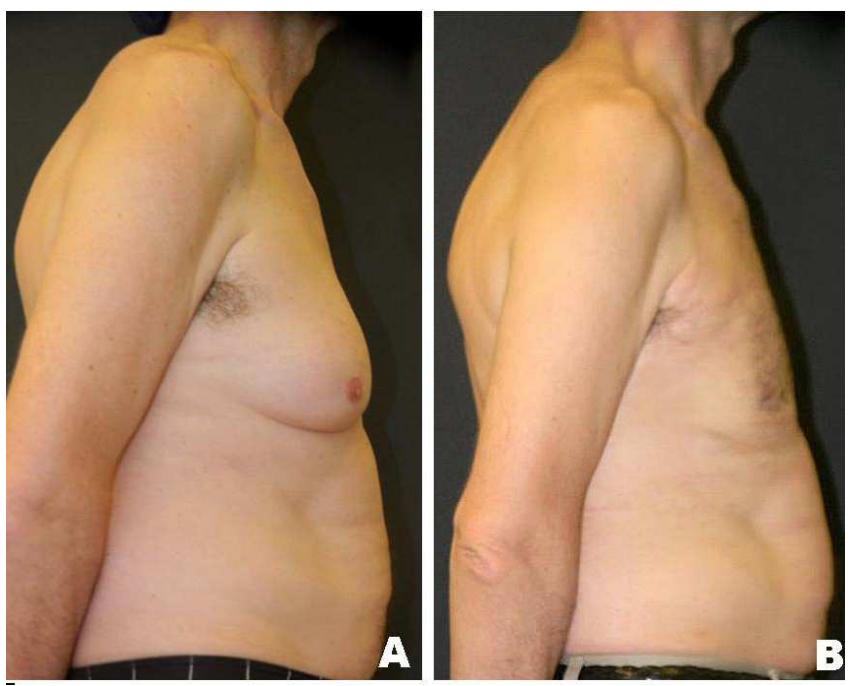

Figura 4 - El mismo paciente de las figuras 3 en visión lateral. A antes y $B$, seis meses después. El cambio de la proyección de la mama es notable, así como la retracción cutánea.

combinación de cirugía y liposucción asistida, realizada por primera vez por Teimourian et al. ${ }^{16}$, supuso importantes avances ${ }^{17,18}$. Una amplia gama de láseres han sido empleados como los de Nd:YAG ${ }^{19}$ y el láser de Diodo ${ }^{20}$. Este último, de emisión en los 980nm, es un sistema altamente eficaz porque emite altas dosis de energía y su tamaño y diseño es práctico y de relativo poco coste, si se le compara con otros sistemas láser.

En nuestro estudio prospectivo de 32 pacientes con ginecomastia, la técnica descrita es segura y reproducible, con un postoperatorio en el que no se observan incidentes en prácticamente ninguno de los pacientes. La valoración subjetiva de los resultados a los seis meses por los pacientes fue, Muy Bueno en 23 pacientes; Bueno en siete, y Regular en dos, y no se observó ningún resultado Malo. En la última valoración a los seis meses, la reducción del perímetro del tórax tuvo un promedio de $12,5 \mathrm{~cm}$, junto a la disminución del diámetro de las areolas. La valoración final de las fotografías para la valoración objetiva por los cirujanos fue, Muy Bueno, 26 casos; Bueno, cinco casos, y Regular en un caso.

Las conclusiones son que la lipólisis asistida por láser obtiene un beneficio estético adicional debido a la contracción de la piel, como consecuencia del efecto térmico residual que deposita la energía láser en el tejido durante el tratamiento. La irradiación láser rompe la membrana del adipocito por efecto térmico al absorber la energía del láser. La lipólisis láser provoca licuefacción de la grasa por acción térmica pero sin que ocurra vaporización ${ }^{21,22}$, debido a que la lisis del adipocito se inicia a una temperatura menor que la necesaria para conseguir su vaporización, no obstante, el efecto térmico consigue coagular los vasos. Este particular hace que el tratamiento de lipólisis láser sea de ayuda de la cirugía y, por lo tanto, se puede recomendar como un buen complemento de la liposucción.

La energía láser acumulada en el área quirúrgica sirve como referencia para repetir la intervención de forma estandarizada, lo que convierte a esta técnica en un procedimiento quirúrgico repetible. Los $8 \mathrm{~kJ}$ a $12 \mathrm{~kJ}$ totales de energía láser acumulada en cada mama de forma homogénea es un punto esencial de referencia, a fin de que esta técnica pueda emplearse por otros sistemas que efectúan la lipólisis con láseres de distintas longitudes de onda. Otros sistemas combinan durante la lipólisis diferentes láseres, siguiendo el principio de una mayor o menor especificidad de la absorción de la energía térmica por el tejido graso.

En la técnica láser empleada en este estudio, al irradiar en modo continuo se cierran los pequeños vasos reduciendo eficazmente el sangrado ${ }^{23}$. El tensado y la firmeza de la piel suceden por coagulación de las fibras de colágeno y como directo resultado del efecto térmico durante la actuación del láser. La piel sigue un lento proceso de recuperación de la flacidez, por lo que este efecto no debería juzgarse antes de dos meses después de la cirugía. Aunque el proceso de recuperación de tejido continúa durante varias semanas después del tratamiento láser ${ }^{24}$, la formación de nuevas fibras de colágeno puede también llevar varios meses. El tensado de las fibras de colágeno, con gran probabilidad, es responsable de la remodelación y corrección de la flacidez cutánea ${ }^{25,26}$. Otros autores señalan que más del $25 \%$ de los casos en los que se había obtenido una reducción adecuada del volumen necesitaron escisión quirúrgica para la flacidez de la piel27,28. En nuestro trabajo, sólo en el 6,25\% de los casos no se obtuvo la retracción de la piel buscada. En consideración de estas cifras. Podríamos afirmar que el tratamiento de la ginecomastia asistida por láser reduce en más de cuatro veces la necesidad de escisión quirúrgica. Estas cifras son alentadoras para el procedimiento quirúrgico con láser.

En base a la casuística presentada, y según hemos observado, los drenajes no son necesarios pero si la colocación de un vendaje compresivo para evitar la acumulación de exudado y la posibilidad de formación de seromas $^{12}$. La reanudación de las actividades normales por parte del paciente es rápida después de la cirugía, lo que convierte a este procedimiento en muy satisfactorio, tanto para el cirujano como para el paciente ${ }^{29}$.

De acuerdo a las observaciones de este estudio, la liposucción asistida por láser como cirugía combinada puede realizarse tanto si el objetivo es eliminar la grasa y el tejido fibrótico subyacente a la glándula mamaria, o para resolver la flacidez cutánea o el excedente de piel, que usualmente debe eliminarse y que exige rediseñar el aspecto pectoral.

Cuando la ginecomastia es voluminosa y la mama es dura a la palpación, las dificultades para eliminar su proyección suelen ser mayores al practicar una liposucción convencional. En estos casos la mastectomía 
subcutánea se indica como el tratamiento de elección ${ }^{30}$, pero, empleando complementariamente el láser de Diodo de $980 \mathrm{~nm}$, la cúpula de la glándula y el tejido fibroso se eliminan de forma satisfactoria, reduciendo su proyección y evitando que, tras la cirugía, la región pectoral presente unas mamas de aspecto puberal femenino.

La liposucción asistida por láser es sencilla de ejecutar si se conocen los principios de la técnica quirúrgica y los fundamentos de la interacción láser-tejido. En nuestra experiencia, la lipólisis láser es efectiva y consigue reducir el diámetro de la areola, tensa la piel y mejora el aspecto flácido del área pectoral. En el polo inferior las maniobras de lipólisis láser es recomendable que excedan la línea inframamaria en $2 \mathrm{~cm}$ o $3 \mathrm{~cm}$, a fin de evitar la sombra proyectada sobre el pecho por el pliegue de la piel. Este detalle evita la apariencia de excesivo volumen residual y la forma propia de una proyección pectoral femenina. Asimismo, el control de la temperatura es crucial durante la actuación del láser, a fin de evitar quemar y lesionar la piel.

\title{
A B S T R A C T
}

\begin{abstract}
Objective: To evaluate efficacy of laser lipolysis in the treatment of gynecomastia to correct breast volume, flaccidity and excess skin without its excision. Methods: Prospectively, 32 patients with gynecomastia under tumescent anaesthesia and sedation underwent laser lipolysis with 980 nm diode laser, 15W continuous emission and 8 to $12 \mathrm{~kJ}$ energy per breast. Externally cold air was used to protect the skin. No drainages were used but a compressive bandage. Patients evaluated results on a VAS scale. Two doctors evaluated results comparing before and 6 month after photographs and also measured the areola and chest diameter. Results: Twenty three patients considered results as Very Good, 7 Good and 2 Fair Cutaneous retraction of the areola was noticeable one month after the surgery and was maximum 6 months after. Evaluation by doctors was 26 Very Good, 5 Good and 1 Fair. There were no burns, ischemia or lesions in areolas or nipples. Conclusion: Laser assisted liposuction is a simple and efficacious technique, barely traumatic and permits a rapid reincorporation to normal activities:
\end{abstract}

Key words: Lasers. Lipolysis. Lasers, semiconductor. Lipectomy. Gynecomastia.

\section{REFERENCIAS}

1. Aegineta P. The Seven books of Paulus Aegineta. London: Sydenham Society; 1847. Traduzido por Francis Adams.

2. Gikas P, Mokbel K. Management of gynecomastia: an update. Int J Clin Pract. 2007;61(7):1209-5.

3. Fabié-Boulard A, Fabre G, Gangloff D, Grolleau JL, Chavoin JP. Gynécomastie. EMC. 2006:1-7.

4. Rohrich RJ, Ha RY, Kenkel JM, Adams Jr WP. Classification and management of gynecomastia: defining the role of ultrasoundassisted liposuction. Plast Reconstr Surg. 2003;111(2):909-23.

5. Weinzweig J. Secretos de la cirugía plástica, reconstructiva y estética. Philadelphia: McGraw-Hill Interamericana; 1999.

6. Simon BE, Hoffman S, Khan S. Classification and surgical correction of gynecomastia. Plast Reconstr Surg. 1973;51(1):48-52.

7. Braunstein GD. Gynecomastia. N Engl J Med. 1993;328(7):490-5.

8. Huang TT, Hidalgo JE, Lewis SR. A circumareaolar approach in surgical management of gynecomastia. Plast Reconstr Surg. 1982;69(1):35-40.

9. Montiel-Jarquín A, Reyes-Páramo P, Ramos-Alvarez G, LópezColombo A, Tinajero-Esquivel M, Ruiz-León B. External periareolar incision for subdermal mastectomy in men with gynecomastia. Cir Cir. 2007;75(5):327-31

10. Apfelberg DB. Results of multicenter study of laser-assisted liposuction. Clin Plast Surg. 1996;23(4):713-9.

11. Apfelberg DB, Rosenthal S, Hunstad JP, Achauer B, Fodor PB. Progress report on multicenter study of laser-assisted liposuction. Aesthetic Plast Surg. 1994;18(3):259-64.

12. Trelles MA, Mordon SR, Bonanad E, Moreno Moraga J, Heckmann A, Unglaub F, et al. Laser-assisted lipolysis in the treatment of gynecomastia: a prospective study in 28 patients. Laser Med Sci. 2012 Feb 19. [Epub ahead of print]

13. Tallón-Aguilar L, Serrano-Borrero I, López-Porras M, SousaVaquero JM, de León-Carrillo J. Breast cancer in males. Cir Cir. 2011;79(4):296-8
14. Klein JA. Tumescent technique for local anesthesia improves safety in large-volume liposuction. Plast Reconstr Surg. 1993;92(6):108598.

15. Goldman A, Schavelzon DE, Blugerman GS. Laserlipólise: lipoaspiração com Nd-YAG laser. Rev Soc Bras Cir Plast. 2002;17(1):17-26

16. Teimourian B, Perman R. Surgery for gynecomastia. Aesthetic Plast Surg. 1983;7(3):155-7.

17. Cook Jr WR. Laser neck and jowl liposculpture including platysma laser resurfacing, dermal laser resurfacing, and vaporization of subcutaneous fat. Dermatol Surg. 1997;23(12):1143-8.

18. Prado A, Andrades P, Danilla S, Leniz P, Castillo P, Gaete F. A prospective, randomized, double-blind, controlled clinical trial comparing laser-assisted lipoplasty with suction-assisted lipoplasty. Plast Reconstr Surg. 2006;118(4):1032-45.

19. Kim KH, Geronemus RG. Laser lipolysis using a novel 1,064 nm Nd:YAG Laser. Dermatol Surg. 2006;32(2):241-8.

20. Reynaud JP, Skibinski M, Wassmer B, Rochon P, Mordon S. Lipolysis using a 980-nm diode laser: a retrospective analysis of 534 procedures. Aesthetic Plast Surg. 2009;33(1):28-36.

21. Mordon S, Eymard-Maurin AF, Wassmer B, Ringot J. Histologic evaluation of laser lipólisis: pulsed 1064-nm Nd:YAG laser versus CW 980-nm diode laser. Aesthetic Surg J. 2007;27(3):263-8.

22. Mordon SR, Wassmer B, Reynaud JP, Zemmouri J. Mathematical modelling of laser lipólisis. Biomed Eng Online. 2008;7:10.

23. Trelles MA, Pardo L, Rigau J, Garcia L. Rapid healing after skin laser resurfacing: a minimal mechanical trauma technique. Ann Plast Surg. 1998;41(3):332-4.

24. Trelles MA, Brychta P, Stanek J, Allones I, Alvarez J, Koegler G, et al. Laser techniques associated with facial aesthetic and reparative surgery. Facial Plast Surg. 2005;21(2):83-98.

25. Trelles MA, Rigau J, Pardo L, García-Solana L, Vélez M. Electron microscopy comparison of $\mathrm{CO} 2$ laser flash scanning and pulse technology one year after skin resurfacing. Int J Dermat. 1999:38(1):58-64. 
26. Goldman A. Submental Nd:Yag laser-assisted liposuction. Lasers Surg Med. 2006;38(3):181-4

27. Hodgson EL, Fruhstorfer BH, Malata CM. Ultrasonic liposuction in the treatment of gynecomastia. Plast Reconstr Surg. 2005;116(2):646-53.

28. Petty PM, Solomon M, Buchel EW, Tran NV. Gynecomastia: evolving paradigm of management and comparison of techniques. Plast Reconstr Surg. 2010;125(5):1301-8.

29. Moreno-Moraga J, Royo J. Our experience treating 500 lipodystrophic areas with a 924/975 nm laser, a new device that induces lipolysis and heating of the dermis and fibrous septa of fatty tissue. J Clin Dermatol [online]. 2010. Disponível em: http:// www.palomarmedical.com/uploaddocs/our-experience-treating500-lipodystrophic-areas-with-a-924-975-nm-laser-a-newdevise-that-induces-lipolysis-and-heating-of-the-dermis-andfibrous-s.pdf.

30. Mordon S, Capon A, Creusy C, Fleurisse L, Buys B, Faucheux M, et al. In vivo experimental evaluation of skin remodeling by using an Er:Glass laser with contact cooling. Lasers Surg Med. 2000;27(1):1-9.
Recebido em 02/06/2012

Aceito para publicação em 01/08/2012

Conflito de interesse: nenhum

Fonte de financiamento: nenhuma

Como citar este artigo:

Trelles M, Bonanad E, Moreno-Moraga J, Alcolea J, Mordon S, Leclére FM. Lipólisis laser y lipossucción en ginecomastia: retracción cutânea eficaz y segura. Rev Col Bras Cir. [periódico na Internet] 2013;40(1). Disponível em URL: http://www.scielo.br/rcbc

Endereço para correspondência:

Franck Marie Leclère

E-mail: franckleclere@yahoo.fr; franck.leclere@inserm.fr 